\title{
Commentary: systematic review and meta-analysis of genetic association studies of pelvic organ prolapse
}

\author{
Mittal Patel ${ }^{1}$ \\ Received: 7 April 2021 / Accepted: 26 April 2021 / Published online: 14 May 2021 \\ (C) The International Urogynecological Association 2021
}

Pelvic organ prolapse (POP) is a common and multifactorial condition. Large population-, family- and twins-based studies have confirmed a genetic risk factor. This fascinating systematic review and meta-analysis by Allen-Brady et al. [1], is aimed at identifying the genetic loci and specific polymorphisms associated with POP, while assessing the strength, consistency and risk of bias amongst reported associations. It is important because the genetic contribution to POP is poorly understood whereas there is string evidence of heritability for POP.

Various studies, including case-control and cross-sectional designs, were included using PubMed and HuGE Navigator, and relevant conference abstracts were searched using genetic and phenotype keywords from 2015 to 2020 . A total of 53 studies (46 published and 7 unpublished) were included and fixed and random effects meta-analyses were conducted using co-dominant models of inheritance. The credibility of pooled associations was assessed using the interim Venice criteria. Four significant polymorphisms in the genes associated with pelvic organ prolapse were found: rs2228480 at the ESR1 gene (OR $0.6795 \%$ CI $0.46-0.98, \mathrm{I}^{2}=0.0 \%$, Venice Rating $\mathrm{BAB}$ ), rs 12589592 at the FBLN5 gene (OR $1.4695 \% \mathrm{CI}$ $1.11-1.82, \mathrm{I}^{2}=36.3 \%$, Venice Rating BBB), rs 484389 at the $P G R$ gene (OR $0.6195 \%$ CI $0.39-0.96, \mathrm{I}^{2}=32.4 \%$, Venice Rating CBB), and rs 1800012 at the COL1A1 gene (OR 0.80 95\% CI 0.66-0.96, $\mathrm{I}^{2}=0.0 \%$, Venice Rating BAB). Genomewide association studies were also considered for metaanalyses and identified further novel variants in different populations. This review is extensive and comprehensive, working from genotype or allele frequencies, rather than using precalculated effect sizes.

This review provides a starting point for consideration of genetic predisposition to POP by highlighting key gene polymorphisms associated with POP. However, extensive work is needed to establish the relevance in clinical practice to improve the management of POP prevention, treatment and patient counselling. The role of genetic disposition in the pathogenesis of POP needs to be established and validated in a variety of different populations. Following this, the possible role of genetic testing in clinical practice needs to be thoroughly evaluated before we can consider the application of genetic testing for developing risk models. This is particularly important when there are multiple dynamic risk factors associated with POP that may play a greater role in symptomatology and quality of life.

\section{Reference}

1. Allen-Brady K, Chua JWF, Cuffolo R, Koch M, Sorrentino F, Cartwright R. Systematic review and meta-analysis of genetic association studies of pelvic organ prolapse. Int Urogynecol J. 2021. https://doi.org/10.1007/s00192-021-04782-2.

Publisher's note Springer Nature remains neutral with regard to jurisdictional claims in published maps and institutional affiliations.
Mittal Patel

m.patel6@nhs.net

1 St Mary's Hospital, Imperial College Healthcare NHS Trust, London, UK 